\title{
Perinatal Central Nervous System Depression
}

National Cancer Institute

\section{Source}

National Cancer Institute. Perinatal Central Nervous System Depression. NCI Thesaurus.

Code C101271.

Generalized depression of the central nervous system of an infant during the perinatal period. 